\title{
3'-daidzein sulfonate protects myocardial cells from hypoxic-ischemic injury via the NRF2/HO-1 signaling pathway
}

\author{
Xueliang Zeng ${ }^{1}$, Junjian Yu', Taohui Zeng ${ }^{1}$, Yuan Liu ${ }^{1}$, Bei Li ${ }^{1}$ \\ ${ }^{1}$ Department of pharmacy, First Affiliated Hospital of Gannan Medical University, Ganzhou, China; ${ }^{2}$ Cardiovascular and Thoracic Surgery \\ Department 2, First Affiliated Hospital of Gannan Medical University, Ganzhou, China \\ Contributions: (I) Conception and design: X Zeng; (II) Administrative support: B Li; (III) Provision of study materials: X Zeng, B Li; (IV) Collection \\ and assembly of data: J Yu, P Liu; (V) Data analysis and interpretation: Y Liu, T Zeng; (VI) Manuscript writing: All authors; (VII) Final approval of \\ manuscript: All authors. \\ Correspondence to: Bei Li. Department of Pharmacy, First Affiliated Hospital of Gannan Medical University, Ganzhou 341000, China. \\ Email: gyfylibei2009@163.com.
}

Background: Myocardial infarction (MI) has a high mortality and disability rate and greatly affects human health. This study sought to explore the therapeutic effect and molecular mechanism of 3'-daidzein sulfonate (DSS) on MI.

Methods: A rat MI model was established and low and high doses of DSS were administered to the rats. An in vitro oxygen glucose deprivation model was used to verify the treatment role and mechanism of DSS. The establishment of the rat MI model was confirmed by electrocardiogram. The tissue changes were detected by HE, Masson's trichrome, TUNEL and TTC staining. Cell viability was detected by CCK-8. The viable and dead cells were detected by Calcein-AM/PI. Apoptotic cells, ROS and JC-1 were detected by flow cytometry apoptosis. The level of proteins was detected by western blotting. MDA, SOD and GSH were detected by ELISA.

Results: The results of Hematoxylin and eosin, TUNEL, and Masson staining showed that the myocardial tissue of the MI group was repaired by DSS. The serum levels of cardiac troponin I (CTnI), lactate dehydrogenase $(L D H)$, creatine kinase-MB $(C K-M B)$, and malondialdehyde $(M D A)$ were decreased by DSS, while the serum levels of superoxide dismutase and glutathione were promoted by DSS. The treatment of DSS activated the Nuclear Factor Erythroid 2-Related Factor 2 (NRF-2)/Heme Oxygenase 1 (HO-1) pathway and inhibited the caspase-3 apoptosis pathway. The in vitro experiment showed that DSS greatly restored cell viability and reduced cell apoptosis. DSS also greatly inhibited mitochondrial membrane potential depolarization, reactive oxygen species production, and oxidative stress. The application of the $N R F-2$ inhibitor, $\mathrm{C}_{29} \mathrm{H}_{25} \mathrm{~N}_{3} \mathrm{O}_{4} \mathrm{~S}$ (ML385), greatly inhibited the treatment role of DSS and the NRF-2/HO-1 pathway, and activated the caspase-3 apoptosis pathway.

Conclusions: In conclusion, this study first identified the beneficial role of DSS in MI. DSS protected myocardial cells by activating the $N R F-2 / H O-1$ pathway and inhibiting cell apoptosis. DSS could be used as a novel drug in the treatment of MI.

Keywords: Myocardial infarction (MI); 3'-daidzein sulfonate; $N R F-2 ; H O-1$; apoptosis

Submitted Nov 23, 2021. Accepted for publication Dec 17, 2021.

doi: $10.21037 /$ jtd-21-1909

View this article at: https://dx.doi.org/10.21037/jtd-21-1909

\section{Introduction}

Ischemic heart injury is caused by cardiac vascular stenosis or the blockage of the myocardial tissue ischemia hypoxia injury. It has the characteristics of high morbidity and mortality. Nearly 17.8 million people die each year from the disease (1). Myocardial infarction (MI) is the leading 
cause of death (1). The loss of cardiac muscle cells is an irreversible change that leads to permanent cardiac dysfunction. Apoptosis is a type of programmed cell death characterized by caspase activation (2). Studies have shown that the activation of the apoptotic signaling pathway plays an important role in cardiac injury (3). After myocardial cell apoptosis due to ischemia and hypoxia injury, the dead myocardial cells are replaced by fibroblasts, which eventually leads to cardiac remodeling, ventricular dysfunction, and even the death of patients (3). Thus, research needs to be conducted to establish treatment directions for protecting cardiac myocytes, reducing apoptosis, and improving the vitality of cardiac myocytes (4).

When the blood vessels supplying the heart narrow or infarct, cardiomyocytes produce large amounts of free radicals due to insufficient oxygen and nutrients (5). These free radicals can oxidize and damage the proteins, lipids, and deoxyribonucleic acid inside cells $(6,7)$. This process is referred to as oxidative stress. However, when the level of oxidative stress exceeds the scavenging capacity of superoxide dismutase (SOD) and glutathione $(G S H)$ peroxidase, and reduces GSH in vivo, cardiomyocytes are damaged by free radicals and undergo apoptosis (8). Oxidative stress can aggravate myocardial damage, and reactive oxygen species (ROS) accumulation in myocardial cells leads to mitochondrial dysfunction, which in turn leads to myocardial apoptosis (9).

Nuclear factor erythroid 2-related factor $2(N R F-2)$ is a redox-sensitive intracellular transcription factor that regulates oxidative stress levels and cardiomyocyte homeostasis (10). NRF-2 has been shown to play an important regulatory role in a variety of diseases, such as ischemic heart disease, heart failure, $M I$, atrial fibrillation, and myocarditis. The nucleus activated NRF-2 could induce the expression of many antioxidant enzymes, like catalase, superoxide dismutase (SOD) and glutathione (GSH). Over one million radicals could be wiped out by these enzymes in one second. Heme oxygenase $1(H O-1)$ is also a key downstream gene of $N R F-2$ transcription. Phosphorylated $N R F-2$ could trigger the production of $H O-1$. NRF-2 and $\mathrm{HO}-1$ could reduce lipid and protein oxidation. HO-1 has anti-oxidant, anti-apoptotic, and anti-inflammatory properties, which can enhance the ability of cells to adapt to oxidative stress. Evidence confirms that the NRF/HO-1 axis inhibits the inflammatory reaction and myocardial cell apoptosis (11).

3 '-daidzein sodium sulfonate (DSS) is a chemically modified daidzein. Daidzein is one of the active components of Pueraria root. As a new water-soluble compound, DSS has been shown to reduce brain edema, improve mitochondrial function, and inhibit the expression of inflammatory factors in brain tissue and nerve cell apoptosis. DSS could protect the blood-brain barrier and reduce its permeability. DSS can repair cerebral ischemia-reperfusion injury (12). There is no study clarify the potential roles of DSS in myocardial tissues. Its role in MI is still unknown. Thus, we conducted in vitro and in vivo experiments to clarify the treatment role and mechanism of DSS on MI. We present the following article in accordance with the ARRIVE reporting checklist (available at https://dx.doi. org/10.21037/jtd-21-1909).

\section{Methods}

\section{Rat MI model establishment}

Experiments were performed under a project license (No. LLSC-2020090802) granted by Ethics Committee of The First Affiliated Hospital of Gannan Medical University, in compliance with institutional guidelines for the care and use of animals. We chose female rats, because they are more docile. Forty-eight Wistar rats (female, $250 \pm 25 \mathrm{~g}$, 10 weeks) were obtained from the animal center. The rats were housed in cages (4 per cage), kept under a 12-h day/ night cycle, and had free access to food and water. The rats were divided into the Sham, MI, MI + DSS-Low (L, $1 \mathrm{mg} / \mathrm{kg})$, and $\mathrm{MI}+$ DSS-High $(\mathrm{H}, 2 \mathrm{mg} / \mathrm{kg})$ groups (12 rats/per group). MI was induced by the ligation of the left anterior descending coronary artery as previously reported (13). An electrocardiograph was conducted immediately after modeling. When specific ST segment elevation was observed in electrocardiogram of rats after modeling, it was confirmed that the myocardial infarction model of rats was successfully prepared. The rats were sacrificed $24 \mathrm{~h}$ after modeling, and blood and myocardial tissues were collected.

\section{HE staining}

To observe the myocardial tissue histological changes, hematoxylin and eosin (HE) staining was conducted. The collected tissues were incubated in formaldehyde $(10 \%)$. Next, gradient ethanol dehydration, paraffin embedding, slicing, and HE staining were performed. The images were taken with a light microscope (Leica, Germany).

\section{Masson's trichrome staining}

The heart tissues were dehydrated with sucrose solution 
$(30 \%)$. The tissues were then embedded with TissueTek (Sakura Finetek USA, Inc., USA), and sliced (7 $\mu \mathrm{m})$. Masson's Trichrome Stain kit (Solarbio, China) was used to stain the infarction area. The Masson positive area was red and the collagen fibers were blue. The images were taken by a light microscope (Leica, Germany).

\section{Apoptosis staining}

To detect the apoptotic cells in the myocardial tissues, TUNEL staining was conducted in accordance with the manufacturer's protocol. The TUNEL kit was purchased from Beyotime (China). The tissues were sliced and the sections were dehydrated by gradient ethanol. Next, the slices were incubated with Triton X-100 (10\%) for 1 hour at $37^{\circ} \mathrm{C}$, and TUNEL reaction reagent was added. 4',6-diamidino-2-phenylindole was used to incubate the slices for 3-5 $\mathrm{min}$ at room temperature. After washing the slices, the TUNEL stain images were taken by an inverted fluorescence microscope (Leica, German).

\section{HL-1 cell culture}

The HL-1 cell is a cell line that originated from a mouse atria cell. This cell line was bought from Zeye Biotech (Shanghai, China). The HL-1 cells were maintained in Roswell Park Memorial Institute (RPMI)-1640 to which fetal bovine serum (10\%, Gibco) and penicillinstreptomycin (1\%, Beyotime) were added. The cells were cultured in an incubator under constant humidity $\left(37^{\circ} \mathrm{C}, 5 \%\right.$ carbon dioxide). The concentration of DSS in DSS-L group and DSS-H group were $5 \mu \mathrm{mol} / \mathrm{mL}$ and $10 \mu \mathrm{mol} / \mathrm{mL}$, respectively.

\section{Cell viability detection}

To detect the cell viability of the HL-1 cells in vitro, a cell counting kit-8 (CCK-8) assay was used (Beyotime). On the $3^{\text {rd }}$ day after seeding the cells into the culture wells, $10 \mu \mathrm{L}$ of CCK-8 solution was added to the wells and incubated with the cells for $2 \mathrm{~h}$ at $37^{\circ} \mathrm{C}$. Next, the culture plates were put into the microplate reader, and the optical density of the each well at $450 \mathrm{~nm}$ was recorded.

\section{Calcein-AM/PI viable and dead cells detection}

The viable and dead cells were detected using a CalceinMethyl 4-acetoxybenzoate (Calcein-AM)/Prodium Iodide
(PI) kit (YEASEN, China). The staining was conducted in accordance with the manufacturer's protocol. Briefly, the cells were collected, and the supernatant was discarded. After being rinsed with assay buffer 3 times, the staining reagent $(100 \mu \mathrm{L})$ was added to the cell mixture and incubated for $15 \mathrm{~min}$ at $37^{\circ} \mathrm{C}$. Next, the cell images were taken with an inverted fluorescence microscope (Leica, German).

\section{Annexin V-FITC, $\mathcal{F C}$-1, and ROS flow cytometry apoptosis detection}

The percentage of apoptotic cells was detected by annexin V-fluorescein isothiocyanate (FITC) flow cytometry assays. An annexin V-FITC assay kit was obtained from Beyotime (Shanghai, China). The cells were collected, and the culture medium was discarded after centrifugation. Annexin V-FITC binding reagent $(195 \mu \mathrm{L})$ was used to incubate the cells. Annexin V-FITC $(5 \mu \mathrm{L})$ and propidium iodide $(5 \mu \mathrm{L})$ were added to the mixture, which was incubated for $20 \mathrm{~min}$ in ice. Next, the cells were analyzed by flow cytometry. Similarly, a JC-1 kit (Beyotime, Shanghai, China) was used to detect the mitochondrial membrane potential. The level of intracellular ROS was determined by ROS flow cytometry assays.

\section{$E G C$}

The electrocardiogram (ECG) recording was conducted as previously reported (14). The electrodes were clamped to the right anterior and superior, left anterior, and inferior skin of the chest. The electrodes were then connected to BL-420N Biological function experimental system (Luo Yu Biological Technology Co., LTD). The ECGs were recorded.

\section{TTC staining}

To detect the infarction area of the myocardial tissues, triphenyl tetrazolium chloride (TTC) staining was conducted as previously reported (15). The hearts of the rats were resected and collected. The myocardial tissues were sliced into $1 \mathrm{~mm}$. TTC (1\%) was applied, and the slices were incubated for $20 \mathrm{mins}$ at $37^{\circ} \mathrm{C}$. Next, the slices were fixed with formalin (10\%) for 12 hour at $4^{\circ} \mathrm{C}$. The images were then taken and analyzed.

\section{Western blotting}

To detect the protein expression of NRF2, Histone 3 
(H3), $\beta$-acting, $H O-1$, kelch like $\mathrm{ECH}$ associated protein 1 (Keap1), Bax B-Cell CLL/Lymphoma 2 (Bcl-2), Bcl2 associated $\mathrm{X}$, apoptosis regulator $(B a x)$ and cleaved caspase 3 in the myocardial tissues and cultured HL-1 cells, western blotting was conducted as previously reported $(16,17)$. The tissues and cells were collected and the total proteins were extracted with radioimmunoprecipitation assay buffer. The protein concentrations of the samples were determined by a bicinchoninic acid kit (Thermo Fisher). The samples were loaded separately with sodium dodecyl sulfate-polyacrylamide gel electrophoresis (SDS-PAGE). The proteins of the PAGE were the transferred onto the polyvinylidene fluoride membranes. After blocking, the primary antibodies [NRF2 (1:500), H3 (1:2,000), $\beta$-actin (1:1,000), HO-1 (1:500), Keap1 (1:1,000), Bax (1:500), Bcl$2(1: 1,000)$, and cleaved caspase3 $(1: 2,000)]$ and horseradish peroxidase-conjugated secondary antibody $(1: 1,000$, Abcam, USA). An Enhanced Chemiluminescent (ECL) kit (Beyotime) was used to visualize the blots.

\section{ELISA assay, MDA, SOD and GSH detection}

The levels of cardiac troponin I (CTnI) (My Biosource Co, USA), creatine kinase-MB (CK-MB) (Roche Diagnostic, Germany), lactate dehydrogenase $(L D H)$ (My Biosource Co, USA), SOD, and GSH in the serum of the rats and the culture medium were detected by enzyme-linked immunoassay (ELISA) assay. MDAMDA was detected by a lipid peroxidation $M D A M D A$ assay kit (Sigma-Aldrich, Germany). The levels of $S O D$ and GSH were detected by kits from Nanjing Jiancheng (China).

\section{Statistical analysis}

EXCEL was used to record the collected data. The statistical analysis was conducted with Graphpad Prism 6.0. The differences between two groups were analyzed with the Student's $t$-test. The differences between three groups were analyzed with a one-way analysis of variance, followed by a Turkey assay. A P value $<0.05$ was considered statistically significant.

\section{Results}

\section{The treatment effect of DSS on MI}

To detect the treatment effect of DSS on MI, we first established a rat MI model. To confirm the successful establishment of the model, rat ECGs were performed. The condition of the rats in the different groups before the experiments was all the same. As Figure $1 A$ shows, the ECG waveform of the Sham group was normal, while the ST segment was greatly elevated in the MI group. The data confirmed the establishment of a rat MI model that could be applied in the subsequent experiments.

Next, we detected the treatment effect of DSS on MI. The infarction area was large in the MI group, while there was no infarction area in the Sham group. DSS treatment decreased the infarction area in a dosage dependent manner, such that a high dose of DSS had a better treatment effect (Figure 1B). In relation to the HE stains, we found normal myocardial fibers in the Sham group, but evidently swollen and disordered myocardial fibers in the MI group, indicating myocardial cell apoptosis (Figure 1C).

TUNEL staining was then conducted to examine the apoptotic status of the myocardial tissues. The TUNEL positive area was evidently more increased in the MI group than the Sham group. The application of DSS (at low and high dosages) evidently decreased the TUNEL positive area (Figure 1D). Further, myocardial fibrosis was evidently promoted in the tissue surrounding the infarct area, and myocardial fibrosis was inhibited by DSS in a dose dependent manner (Figure 1E). These results indicate that DSS can decrease the infarction area by inhibiting apoptosis.

\section{DSS decreases hematological indexes, inhibits the caspase-3 apoptosis patbway, and regulates the NRF2/HO-1 axis}

To further clarify the treatment effect and mechanism, the hematological indexes, including $C T n I, C K-M B, L D H$, $M D A, S O D$, and $G S H$, were detected. Compared to the Sham group, the serum levels of $C T n I, C K-M B, L D H$, and $M D A M D A$ were greatly increased in the MI group, while the serum levels of $S O D$ and GSH were evidently decreased. The application of DSS evidently reversed the effect of MI in the levels of these hematological indexes (Table 1).

To clarify the mechanism of DSS in treating MI, the expression of key genes in the NRF2/HO- 1 pathway was detected in the myocardial tissues. As Figure $2 A, 2 B$ show, the nuclear NRF2 was more increased in the MI group than the Sham group. The application of DSS promoted the nuclear translocation of NRF2 in a dose dependent manner. The expression of Keap1 was more decreased in the MI group than the Sham group. The treatment of DSS further decreased the expression of Keap1 in a dose dependent manner. Additionally, DSS inhibited the caspase- 3 apoptosis 

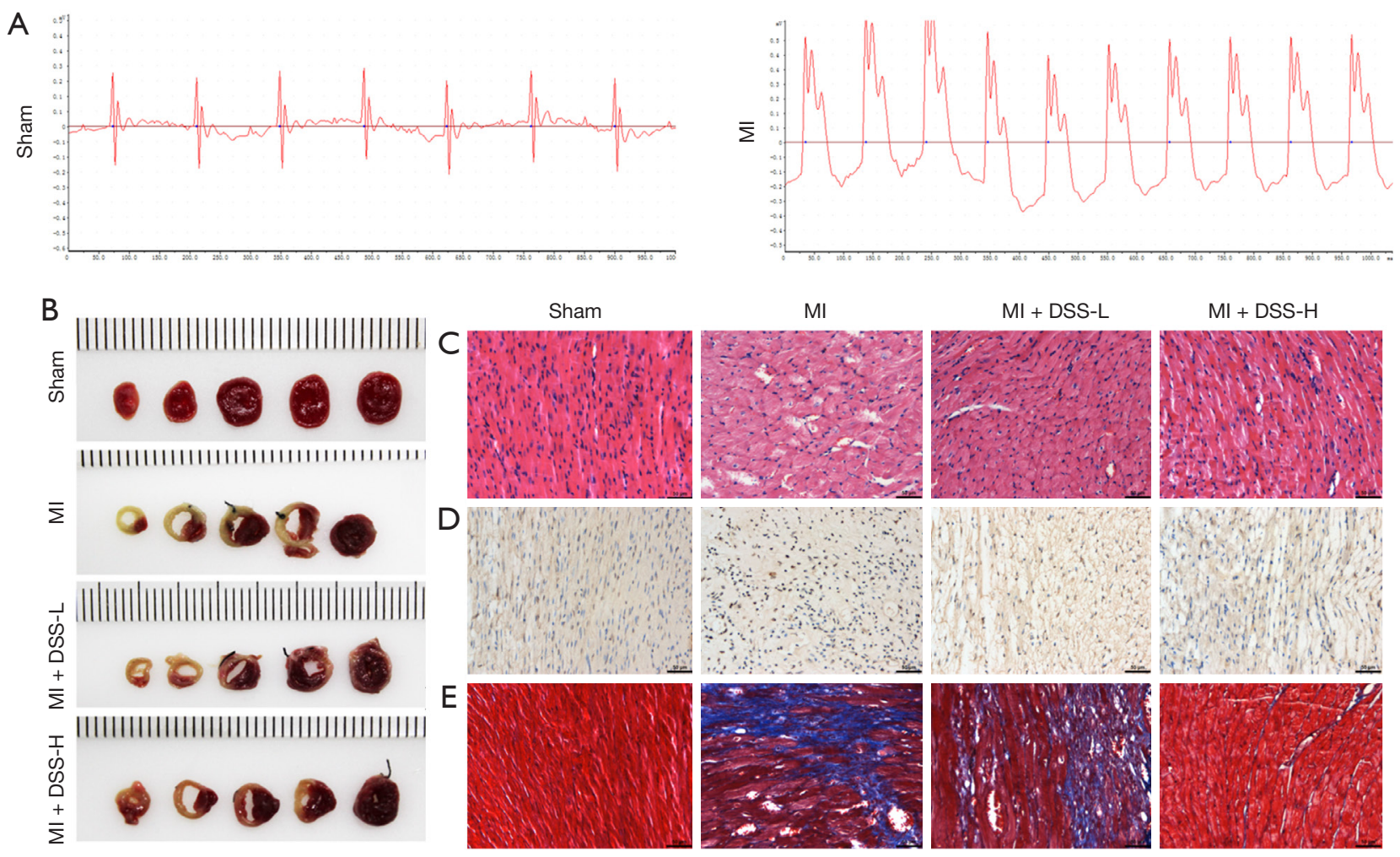

Figure 1 The treatment effect of DSS on MI. (A) ECGs of the Sham and MI group; (B) TTC staining of the Sham, MI, MI + DSS-L, and MI + DSS-H groups; HE staining (C), TUNEL staining (D) and Masson staining (E) of Sham, MI, MI + DSS-L and MI + DSS-H groups $(\mathrm{n}=6, \mathrm{bar}=50 \mu \mathrm{m})$. DSS, 3'-daidzein sodium sulfonate; MI, myocardial infarction; ECGs, electrocardiograph; L, low concentration; H, high concentration; TTC, triphenyltetrazolium chloride; HE, hematoxylin and eosin; TUNEL, TdT-mediated dUTP Nick-End Labeling.

Table 1 The level of CTnI, LDH, CK-MB, MDA, SOD and GSH in rat

\begin{tabular}{|c|c|c|c|c|c|c|}
\hline Group & CTnl (ng/mL) & LDH (U/L) & CK-MB (U/L) & MDA ( $\mu \mathrm{mol} / \mathrm{mg}$ protein) & SOD (U/mg protein) & GSH (U/g protein) \\
\hline Sham & $10.7 \pm 2.3$ & $197.8 \pm 26.3$ & $428.5 \pm 79.4$ & $3.1 \pm 0.3$ & $34.8 \pm 3.9$ & $0.93 \pm 0.10$ \\
\hline MI & $31.4 \pm 4.0$ & $394.2 \pm 35.1$ & $1,001.5 \pm 114.0$ & $13.1 \pm 2.0$ & $20.6 \pm 2.6$ & $0.48 \pm 0.06$ \\
\hline MI + DSS-L & $25.2 \pm 4.8$ & $341.6 \pm 36.9$ & $810.4 \pm 125.9$ & $10.1 \pm 1.9$ & $24.1 \pm 3.0$ & $0.59 \pm 0.06$ \\
\hline $\mathrm{MI}+\mathrm{DSS}-\mathrm{H}$ & $19.6 \pm 5.7$ & $296.9 \pm 23.8$ & $670.1 \pm 103.4$ & $8.0 \pm 2.1$ & $27.5 \pm 3.5$ & $0.74 \pm 0.07$ \\
\hline
\end{tabular}

CTnl, cardiac troponin I; LDH, lactate dehydrogenase; CK-MB, creatine kinase-MB; MDA, malondialdehyde, SOD, superoxide dismutase; GSH, glutathione; DSS, 3'-daidzein sodium sulfonate; L, low concentration; $\mathrm{H}$, high concentration; MI, myocardial infarction

pathway by promoting the expression of $B c l-2$ and inhibiting the expression of Bax and cleaved-caspase-3. These data confirm the role of DSS in regulating the NRF2/HO-1 axis and caspase-3 pathway.

\section{DSS protects myocardial cells in the OGD condition}

To further examine the protective role of DSS in myocardial ischemia (I/S) injury, HL-1 myocardial cells were cultured under an oxygen glucose deprivation (OGD) condition and low and high doses of DSS were administered to treat myocardial cells. As Figure $3 A$ shows, OGD greatly inhibited cell viability, while DSS treatment evidently promoted cell viability in a dose dependent manner. Compared to the Control group, OGD greatly increased the percentage of apoptotic cells, while DSS evidently 
A

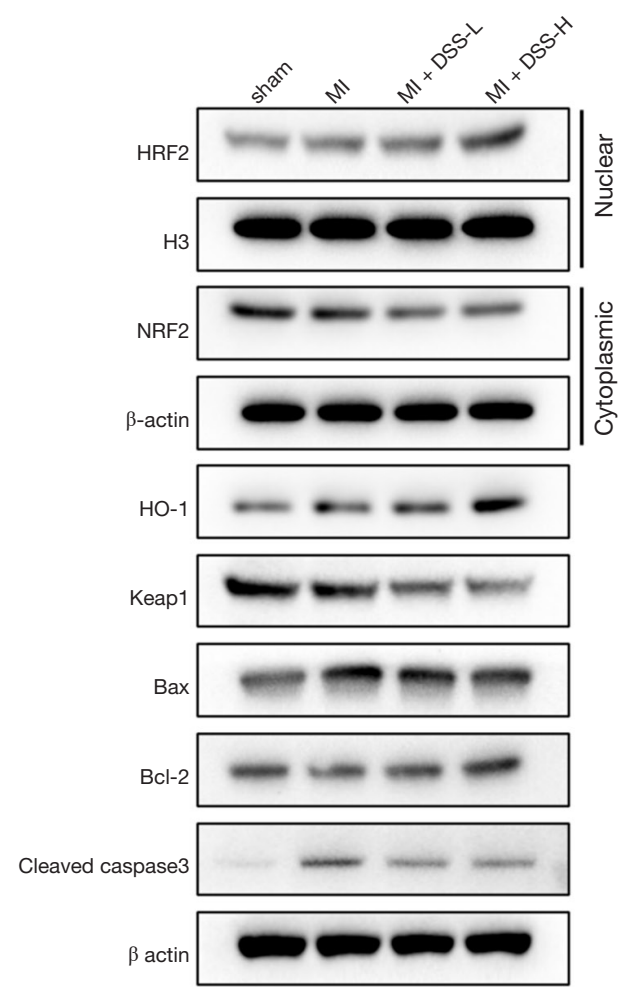

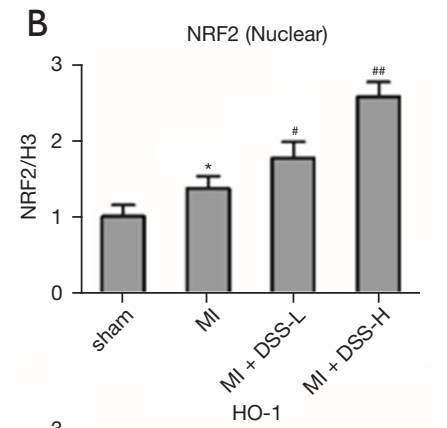
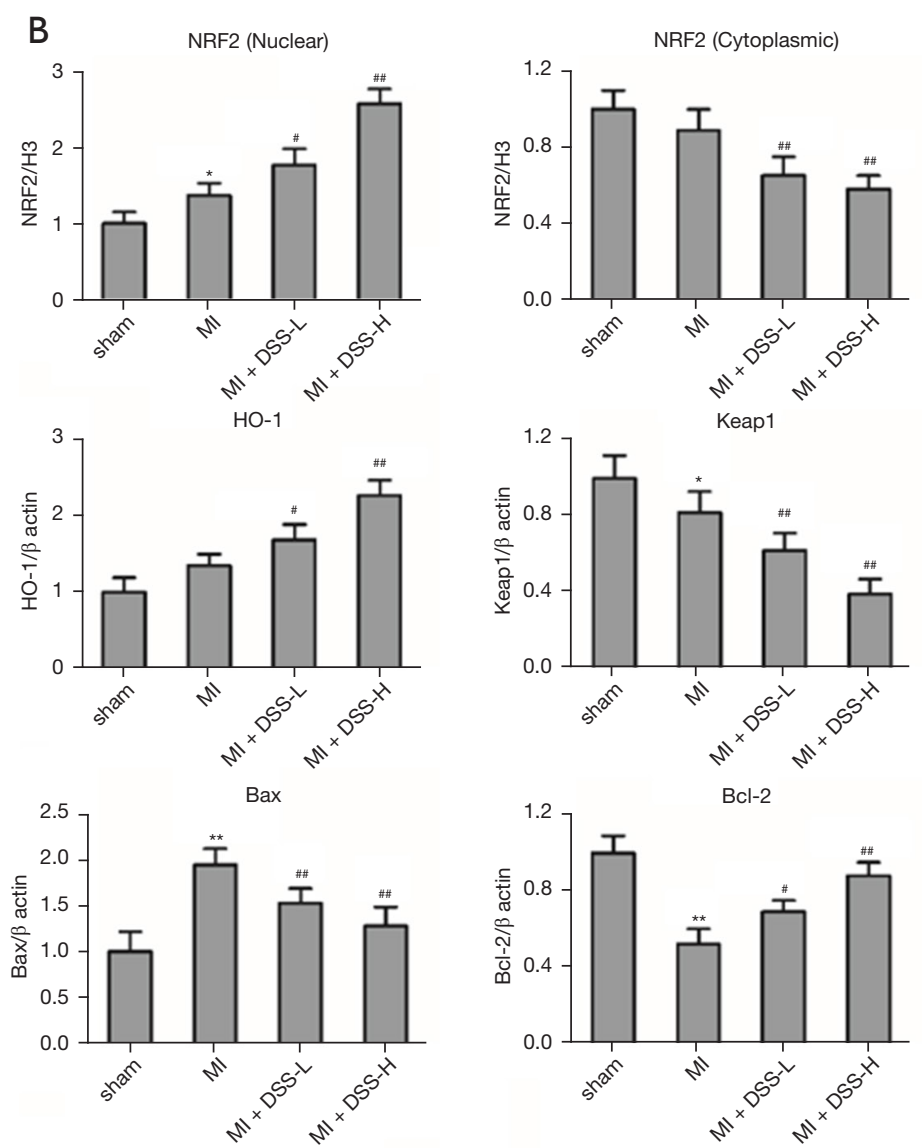

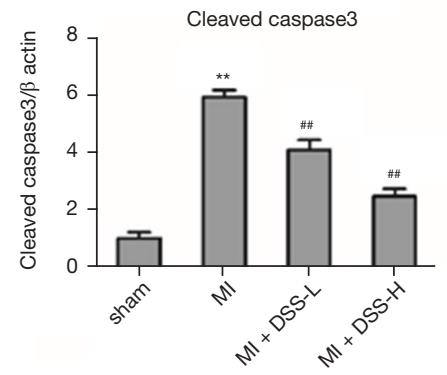

Figure 2 DSS decreases hematological indexes, inhibits the caspase-3 apoptosis pathway, and regulates the NRF2/HO-1 axis. (A) The protein expression of Sham, MI, MI + DSS-L, and MI + DSS-H groups detected by Western Blotting; (B) the histogram of these proteins (MI

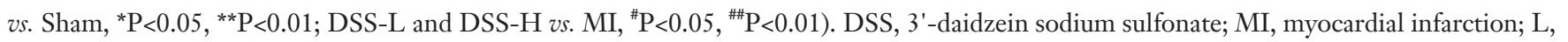
low concentration; $\mathrm{H}$, high concentration.

decreased the percentage of apoptotic cells (Figure 3B). Calcein-AM/PI staining also showed that the death cell number was more increased in the OGD group than the Control group, and the death cell number was reduced in DSS-L and DSS-H groups compared to the OGD group (Figure 3C).

The decrease of mitochondrial membrane potential is a landmark event in the early stage of apoptosis. As the JC-1 flow cytometry assay showed, the mitochondrial membrane potential depolarization was significantly more increased in the OGD group than the Control group. The application of DSS significantly reduced the mitochondrial membrane potential depolarization (Figure 3D). The dysfunction of mitochondria induced by OGD could trigger the production of ROS in cultured HL-1 cells. As Figure $3 E$ and Figure $3 F$ show, fluorescence intensity was greatly induced 
A

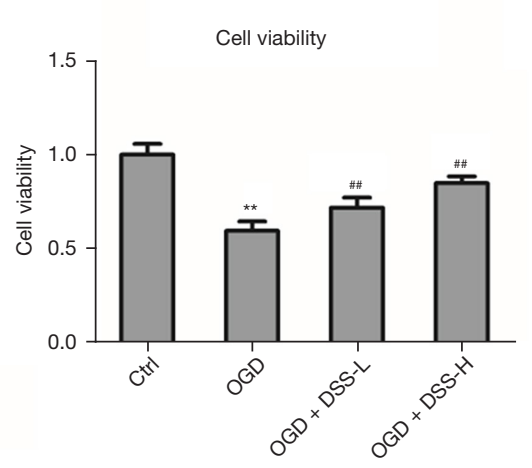

C

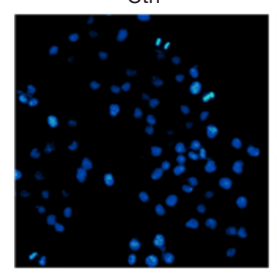

DSS-L

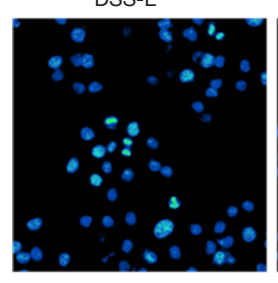

$\mathrm{E}$
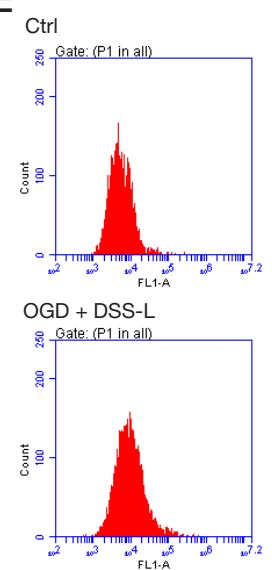

OGD/R

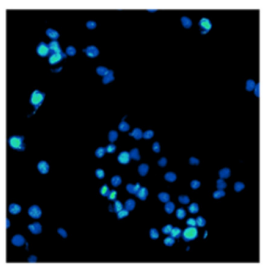

DSS-H

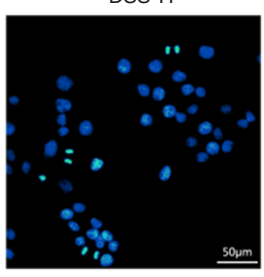

OGD

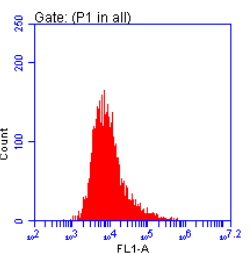

OGD + DSS-H

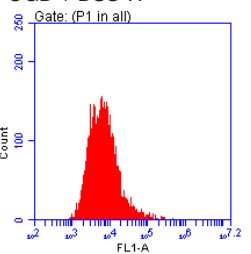

$B^{\mathrm{Ctrl}}$

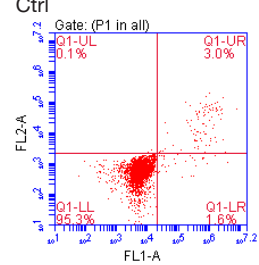

OGD + DSS-L

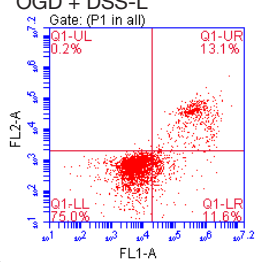

D ctrl

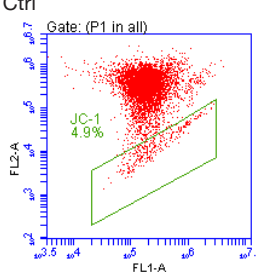

OGD

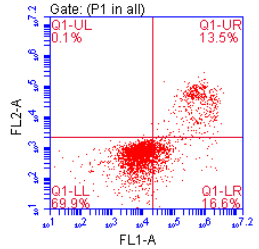

OGD + DSS-H

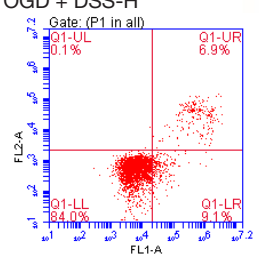

OGD
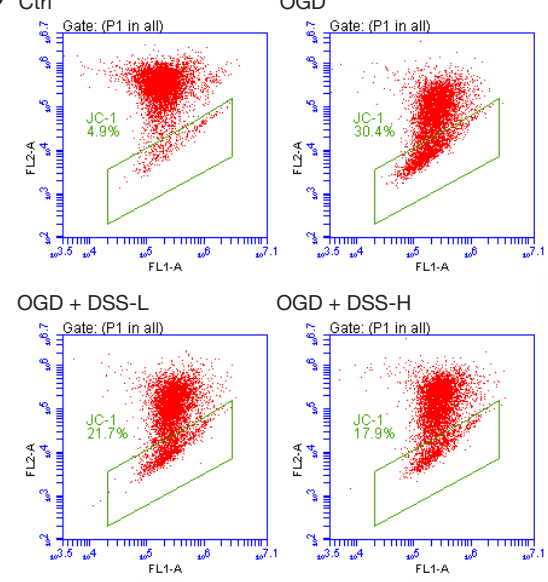

OGD + DSS-H

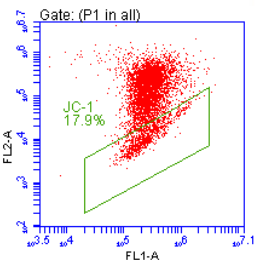

F

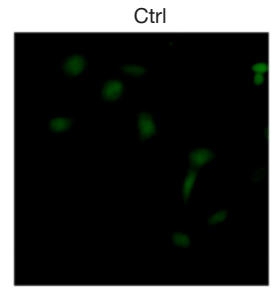

DSS-L

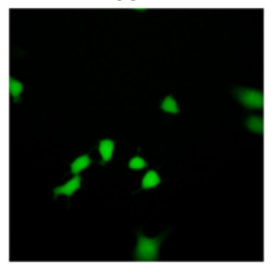

Cell apoptosis
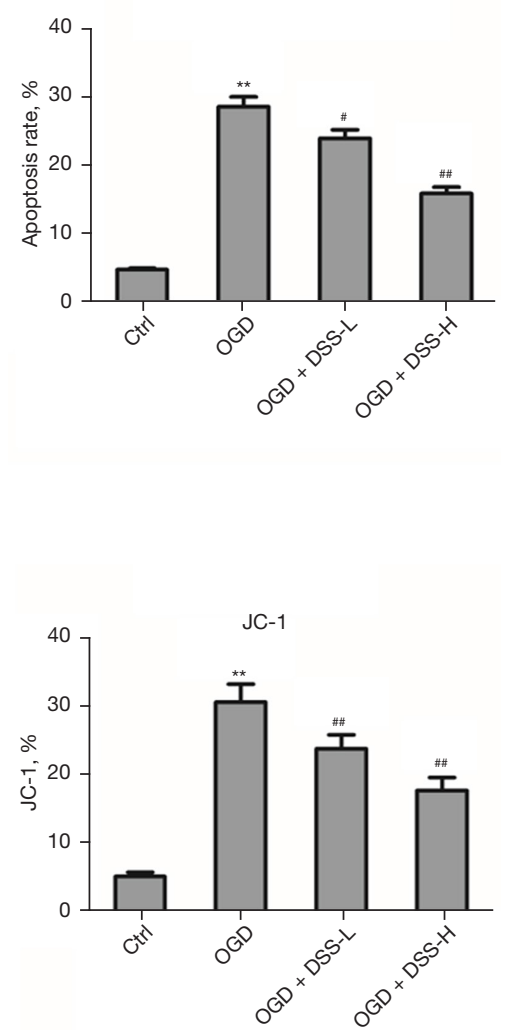

$\mathrm{OGD} / \mathrm{R}$

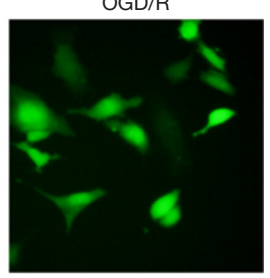

DSS-H

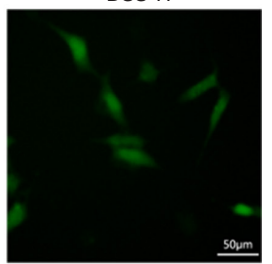

Figure 3 DSS protects myocardial cells in the OGD condition. (A) The cell viability detected by CCK-8; (B) the apoptotic cell percentage detected by Annexin V-FITC flow cytometry; (C) the death cell staining by Calcein-AM/PI; (D) the mitochondrial membrane potential depolarization detected by JC-1 flow cytometry; ROS detection by flow cytometry (E) and fluorescence staining (F) (n=4, OGD vs. Control, ${ }^{* *} \mathrm{P}<0.01$; DSS-L and DSS-H vs. Control, ${ }^{\prime \prime} \mathrm{P}<0.05,{ }^{* \#} \mathrm{P}<0.01$ ). DSS, 3 -daidzein sodium sulfonate; OGD, Oxygen Glucose Deprivation; CCK-8, Cell Counting Kit-8; Calcein-AM/PI, Calcein-Methyl 4-acetoxybenzoate/Prodium Iodide; ROS, reactive oxygen species; L, low concentration; $\mathrm{H}$, high concentration. 
Table 2 The level of MDA and SOD in cultured cells

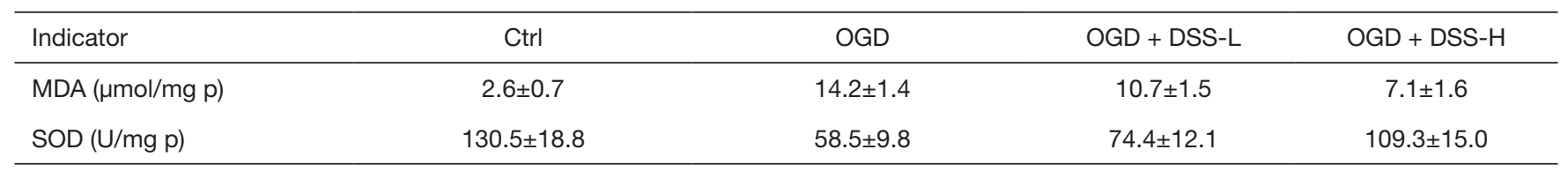

MDA, malondialdehyde; SOD, superoxide dismutase; OGD, oxygen glucose deprivation; DSS, 3'-daidzein sodium sulfonate; L, low concentration; $\mathrm{H}$, high concentration.

by OGD. The promotive role of OGD was evidently inhibited by DSS treatment in a dose dependent manner. As Table 2 shows, the level of $M D A$ was significantly more increased in the OGD group than the Control group. The application of DSS decreased the level of $M D A$ more than the OGD group. These results confirm the cytoprotective role of DSS on myocardial cells in OGD insulation.

\section{DSS decreases SOD and MDA levels, inhibits the caspase-3 apoptosis pathway, and regulates the NRF2/HO-1 axis in vitro}

We also examined whether DSS could regulate the caspase-3 apoptosis pathway and NRF2/HO-1 axis in vitro. To clarify the regulation mechanism of DSS in cultured myocardial cells, the expression of key genes in the NRF2/ HO-1 pathway was detected. As Figure $4 A$ and Figure $4 B$ show, the NRF2 was more increased in the OGD group than the Control group. The application of DSS promoted the nuclear translocation of the NRF2 in a dose dependent manner. The expression of Keap 1 was more decreased in the OGD group than the Control group. The treatment of DSS further decreased the expression of Keap1 in a dose dependent manner. Further, DSS inhibited the caspase- 3 apoptosis pathway by promoting the expression of $\mathrm{Bcl}-2$ and inhibiting the expression of Bax and cleaved-caspase-3. These data confirm the role of DSS in regulating the NRF2/HO-1 axis and caspase-3 pathway in vitro.

\section{The NRF2/HO-1 axis is the main pathway involved in the protection of DSS in myocardial cells}

We confirmed the protective role and the NRF2/HO-1 axis regulation role of DSS. However, we also sought to examine the role of DSS via the NRF2/HO-1 axis. Thus, we applied an NRF2 blocker, ML385, to inhibit the NRF2/HO-1 axis. As Figure $5 \mathrm{~A}$ shows, the cell viability was evidently more inhibited in the OGD group than the Control group. This inhibitive role was greatly reduced by DSS. However,
ML385 greatly inhibited the promotive role of DSS. The percentage of apoptotic cells was more increased in the OGD group than the Control group. This increase was restrained by DSS treatment. However, ML385 evidently inhibited the treatment role of DSS (Figure 5B). CalceinAM/PI staining also showed that ML385 significantly inhibited the cyto-protective role of DSS (Figure 5C). The JC-1 flow cytometry assay showed the mitochondrial membrane potential depolarization was reduced by DSS, and this reduction was inhibited by ML385 (Figure 5D). The production of ROS was also reduced by DSS, but ML385 blocked this reduction (Figure 5E, $5 F$ ). Further, the level of $M D A$, which was inhibited by DSS, was promoted by ML385, while the level of $S O D$, which was promoted by DSS, was inhibited by ML385 (Table 3).

To verify whether the key genes in the caspase-3 apoptosis pathway and NRF2/HO-1 axis were inhibited by ML385, we detected the protein expression of NRF2, Keap1,HO-1, Bax, Bcl-2, and cleaved-caspase-3. The expression of $N R F 2$ in cell nucleus was more increased in the DSS-H group than the OGD group, while ML385 greatly inhibited the role of DSS. The expression trend of NRF2 in the cytoplasm was opposite to that in cell nucleus. The expression of HO-1 and Heap1 in DSS-L and DSS-H groups was more promoted and inhibited compared to the OGD group. Similarly, DSS promoted $B c l-2$ and inhibited Bax and cleaved-caspase-3 expression. However, the application of ML385 reversed the role of DSS (Figure 6A,6B). These results indicate that the NRF2/HO-1 axis is the key pathway involved in the protection of DSS in myocardial cells.

\section{Discussion}

After MI, cardiac function is permanently reduced due to the non-regeneration of myocardial cells (18). Thus, the protection of myocardial cell apoptosis under ischemia and hypoxia exposure is of great clinical significance in the treatment of MI $(19,20)$. Since natural plant ingredients 
A

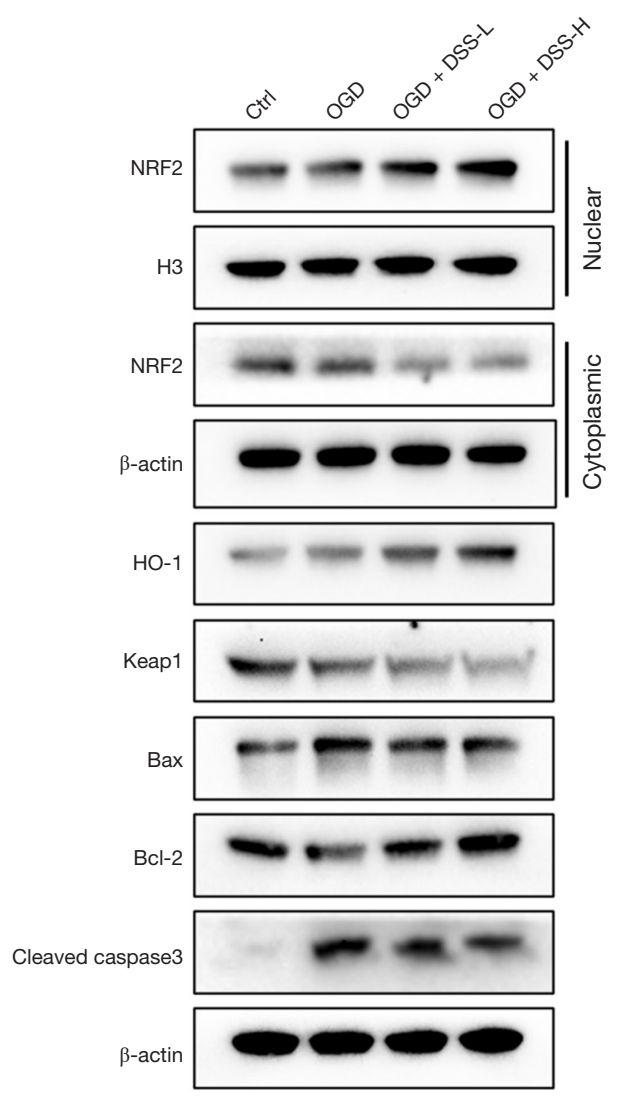

B
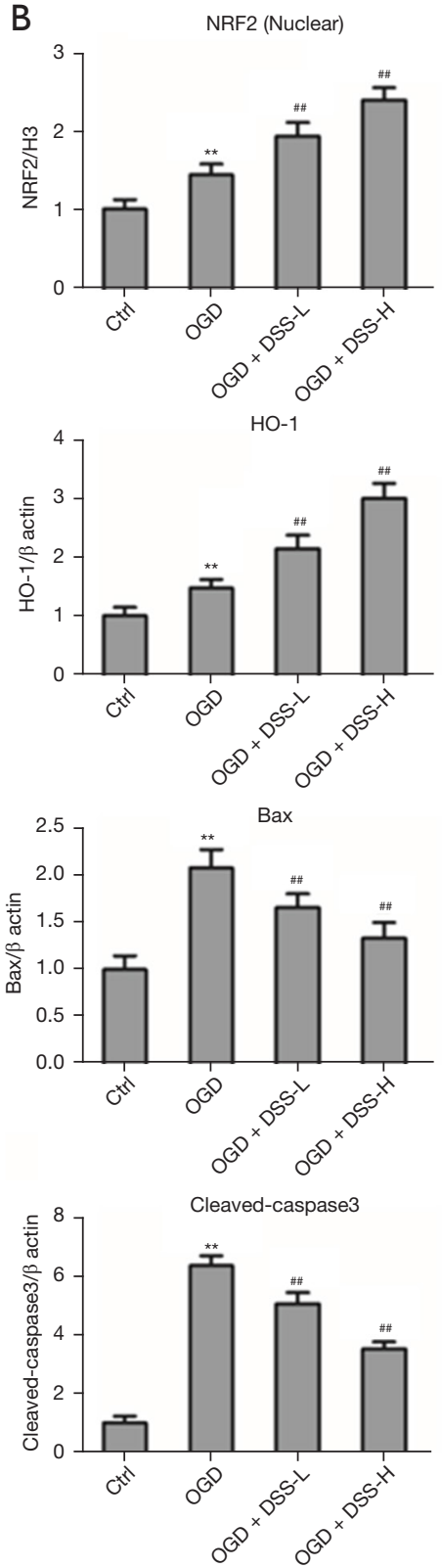
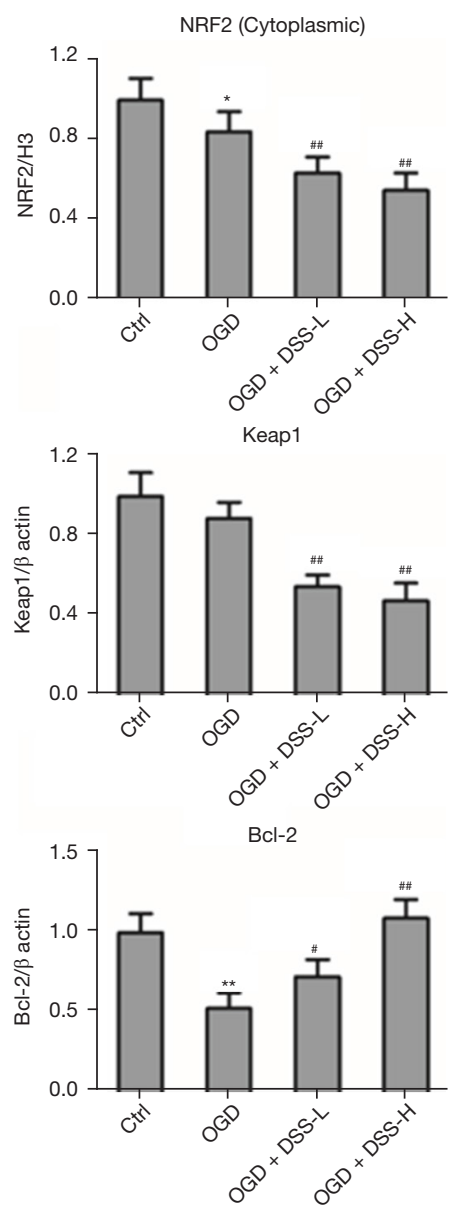

Figure 4 DSS decreases SOD and MDA levels, inhibits the caspase-3 apoptosis pathway, and regulates the NRF2/HO-1 axis in vitro. (A) The protein expression of the Control, OGD, OGD + DSS-L and OGD + DSS-H groups detected by Western Blotting; (B) the histogram of these proteins ( $\mathrm{n}=4$, OGD vs. Control, ${ }^{*} \mathrm{P}<0.05,{ }^{* *} \mathrm{P}<0.01$; DSS-L and DSS-H vs. OGD, ${ }^{*} \mathrm{P}<0.05$, $\left.{ }^{\#} \mathrm{P}<0.01\right)$. DSS, $3{ }^{\prime}-$ daidzein sodium sulfonate; OGD, oxygen glucose deprivation; $S O D$, Superoxide dismutase; $M D A$, malondialdehyde; $N R F 2$, nuclear factor erythroid 2-related factor 2; HO-1, Heme Oxygenase 1; L, low concentration; H, high concentration.

have few side effects, it is of great significance to discover therapeutic drugs for the clinical treatment of MI. DSS is a new water-soluble compound synthesized from daidzein derived from Pueraria root. DSS has been shown to reduce brain edema, improve mitochondrial function, and inhibit the expression of inflammatory factors in brain tissue and nerve cell apoptosis. DSS can repair cerebral ischemiareperfusion injury (12). In this study, we investigated the therapeutic effect of DSS on MI rats.

After MI, myocardial fibers become swollen and 

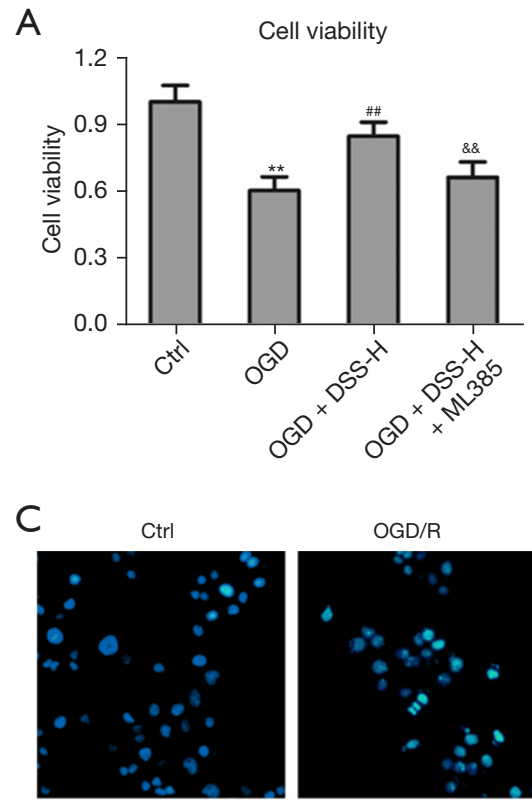

DSS-H

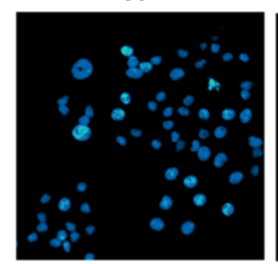

E
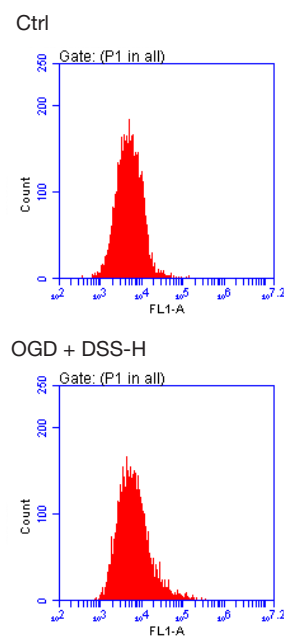

DSS-H + ML385

OGD
B Ctr

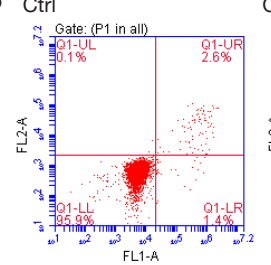

OGD
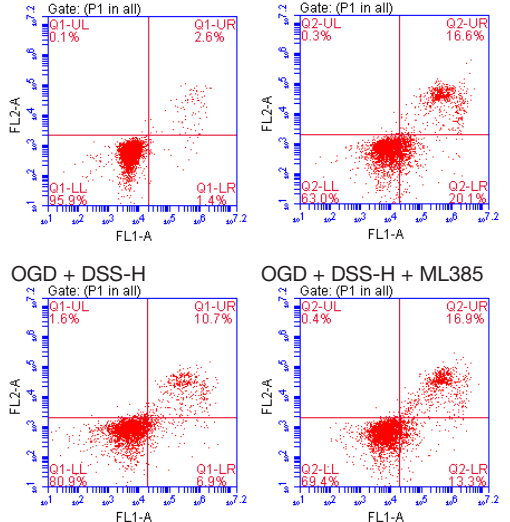

OGD + DSS-H + ML385

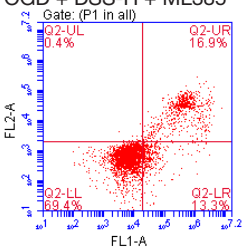

D Ctr
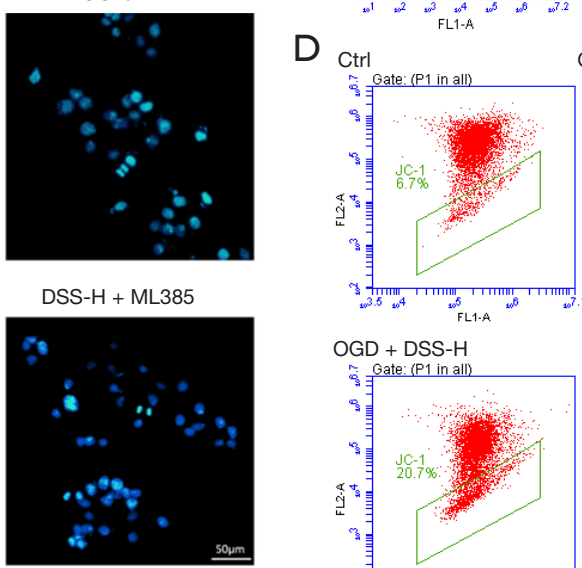

OGD
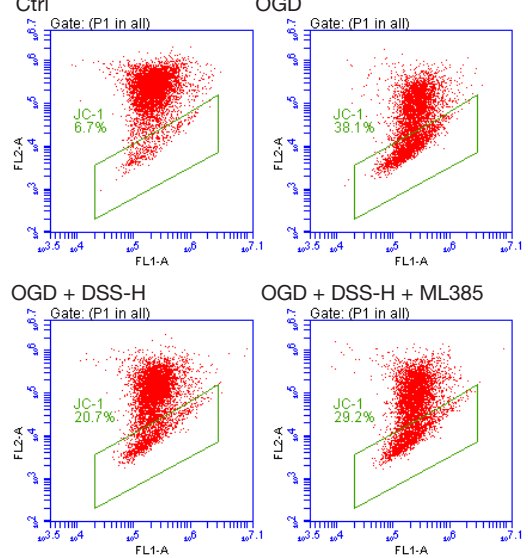

OGD + DSS-H + ML385
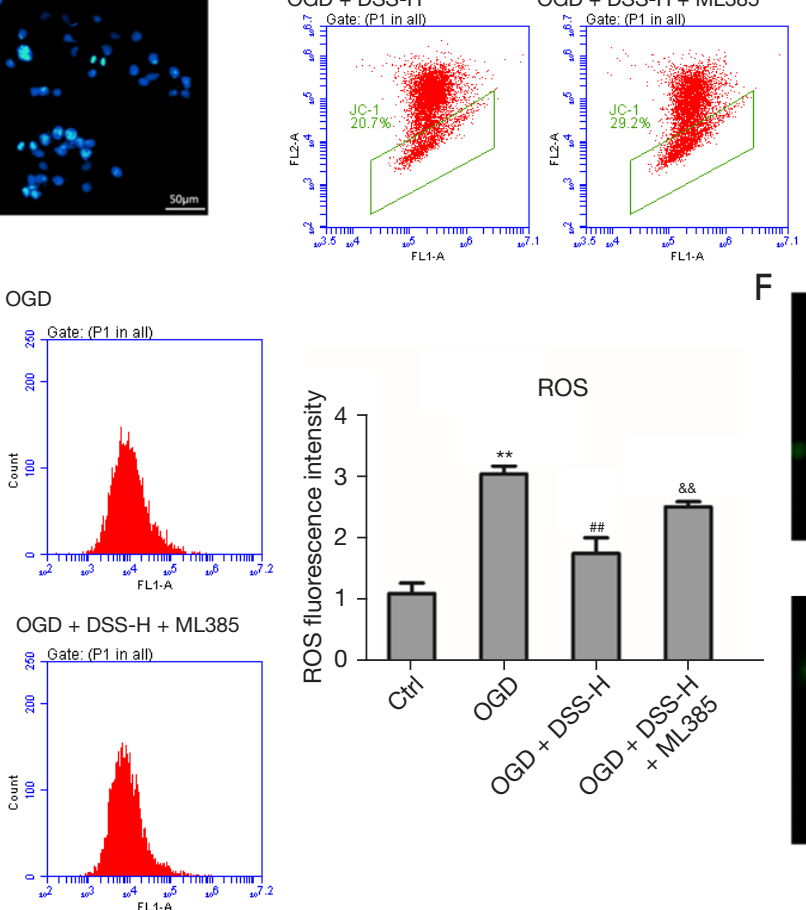

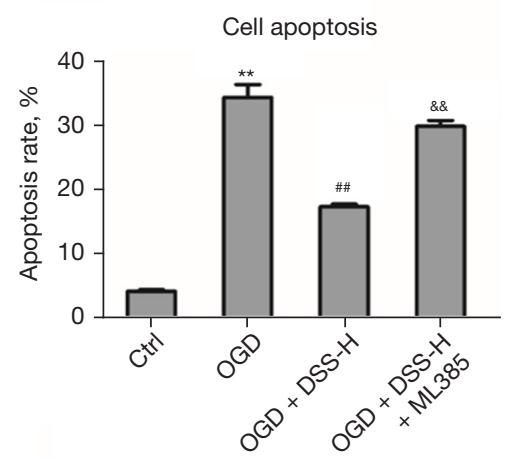

$\mathrm{JC}-1$

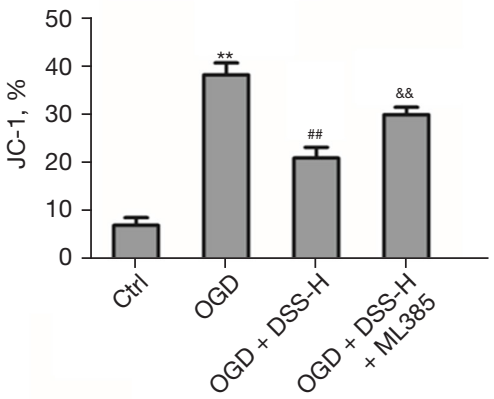

Ctrl

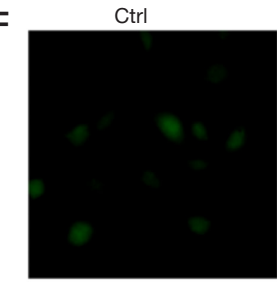

DSS-H

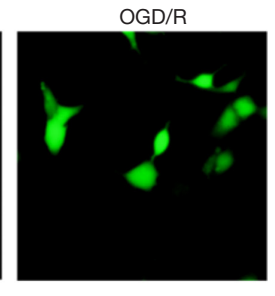

DSS-H + ML385
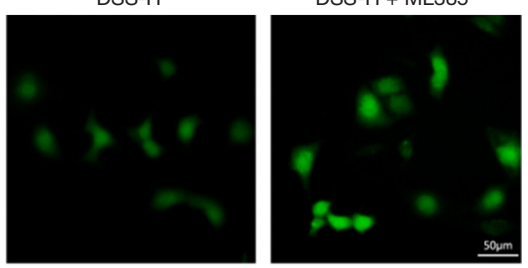

Figure 5 The NRF2/HO-1 axis is the main pathway involved in the protection of DSS in myocardial cells. (A) The cell viability detected by CCK-8; (B) the apoptotic cell percentage detected by Annexin V-FITC flow cytometry; (C) the death cell staining by Calcein-AM/ PI; (D) the mitochondrial membrane potential depolarization detected by JC-1 flow cytometry; ROS detection by flow cytometry (E) and fluorescence staining (F) ( $\mathrm{n}=4$, OGD vs. Control, ${ }^{*} \mathrm{P}<0.01$; DSS-H vs. OGD, ${ }^{\# \#} \mathrm{P}<0.01$; DSS-H + ML385 vs. DSS-H groups, ${ }^{\text {\&\& }} \mathrm{P}<0.01$ ). DSS, 3'-daidzein sodium sulfonate; OGD, oxygen glucose deprivation; NRF2, nuclear factor erythroid 2-related factor 2; HO-1, Heme Oxygenase 1; Calcein-AM/PI, Calcein-Methyl 4-acetoxybenzoate/Prodium Iodide; ROS, reactive oxygen species; L, low concentration; H, high concentration. 
Table 3 The level of MDA and SOD in cultured cells

\begin{tabular}{lcccc}
\hline Indicator & Ctrl & OGD & OGD + DSS-H & OGD + DSS-H + ML385 \\
\hline MDA $(\mu \mathrm{mol} / \mathrm{mg} \mathrm{p})$ & $2.9 \pm 1.5$ & $14.7 \pm 1.1$ & $7.4 \pm 1.0$ & $11.9 \pm 1.7$ \\
SOD $(\mathrm{U} / \mathrm{mg} \mathrm{p})$ & $125.3 \pm 19.8$ & $54.6 \pm 9.3$ & $102.0 \pm 6.9$ & $68.8 \pm 9.4$ \\
\hline
\end{tabular}

MDA, malondialdehyde; SOD, superoxide dismutase; OGD, oxygen glucose deprivation; DSS, 3'-daidzein sodium sulfonate; L, low concentration; $\mathrm{H}$, high concentration.

disorganized, and are infiltrated by inflammatory cells. A large number of cardiomyocytes undergo apoptosis. Apoptotic cardiomyocytes are replaced by fibroblasts and cardiac remodeling occurs, which eventually causes a permanent decline in heart function. In this study, we found that DSS acted in a dose-dependent manner to reduce myocardial apoptosis and the fibroblast area after MI. The detection of MI markers and oxidative stress indicators showed that DSS significantly reduced the levels of $C T n I$, $L D H, C K-M B$, and $M D A$, while restoring the expression levels of $S O D$ and $G S H$. These results suggest that the therapeutic effect of DSS may be due to its ability to regulate cardiomyocytes to resist oxidative stress.

We then examined gene expression in the signaling pathways associated with oxidative stress (NRF2/HO-1) and apoptosis (caspase-3). The NRF-2/HO-1 pathway has been proven to play anti-oxidant, anti-apoptotic, and antiinflammatory roles in cell protection $(21,22)$. The activation of NRF-2/HO-1 has been shown to reduce cardiac injury (23). In this study, we found that DSS promoted the nuclear transfer of $N R F-2$. Keap1 is an E3 ligase, which induces the degradation of $N R F 2$ by the ubiquitin-proteasome system. We also found the expression of Keap-1 was inhibited after DSS treatment. Thus, the upregulation of nuclear $N R F-2$ could be the result of Keap-1 downregulation. The pro-apoptosis genes, Bax and cleaved-caspase-3, were evidently inhibited by DSS, while the anti-apoptosis gene, $B c l-2$, was greatly promoted by DSS. Thus, we found that DSS regulated the NRF/HO-1 pathway and protected myocardial tissues in MI rats.

Cardiomyocytes are the most overworked cells in the body, as the heart needs to beat all the time. Thus, the number of mitochondria in cardiomyocytes has to be very large to meet the energy requirements of cardiomyocytes. However, under the condition of myocardial ischemia and hypoxia, the mitochondrial function of myocardial cells is impaired, which directly affects cardiac function (24). To verify the mechanism of DSS on MI, myocardial cells, HL-1 cells, were cultured in an OGD condition. We found that DSS restored cell viability and reduced the apoptotic cell percentage. Our in vivo experiments showed that oxidative stress levels, as verified by $M D A, S O D$, and $G S H$ detection, in the serum of rats were elevated; thus, we hypothesized that DSS modulates the oxidative stress response of cardiomyocytes by protecting mitochondria. We found the mitochondrial membrane potential depolarization was greatly decreased by DSS. The production of ROS was also greatly reduced by DSS. DSS also inhibited and promoted the level of $M D A M D A$ and $S O D$, respectively.

To verify the oxidative stress regulation mechanism of DSS, we used the NRF-2 inhibitor, ML385, to block the NRF-2/HO-1 pathway. We found that DSS had a treatment role in cell viability, such that the apoptosis and the oxidative stress of myocardial cells were greatly restrained by ML385. The NRF-2, HO-1, was greatly reduced by DSS, while the expression of Keap-1 was reduced by DSS. Further, the pro-apoptotic proteins, Bax and cleaved-caspase-3, and the anti-apoptotic protein, $B c l-2$, were reduced and promoted by DSS, respectively. The pathway regulation role of DSS was greatly reversed by ML385. Thus, we concluded that NRF-2/HO-1 is the key pathway by which DSS treats MI.

In conclusion, in this study, we first examined the treatment role of DSS in MI, and found that DSS inhibited myocardial cell apoptosis and oxidative stress in MI rats. Further, our in vitro experiments confirmed the protective role of DSS in OGD-treated cells. The NRF-2/HO-1 pathway was verified to be the key pathway by which DSS protected myocardial cells. This study verified the application role of DSS in MI. This drug could be a novel therapeutic choice for treating MI.

\section{Acknowledgments}

Funding: The study was funded by the National Natural Science Foundation of China (No. 81160399). 

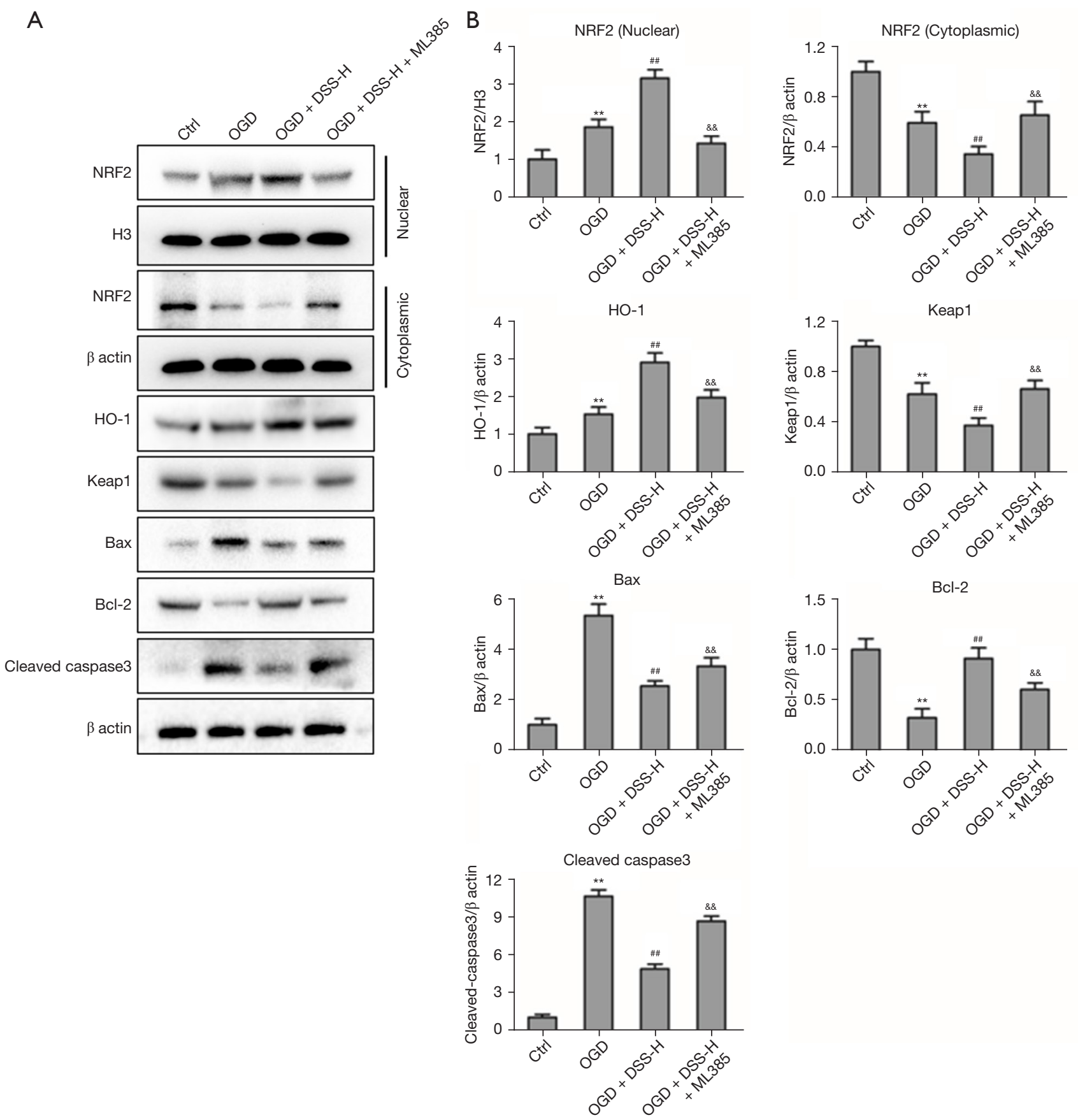

Figure 6 The NRF2/HO-1 axis is the main pathway involved in the protection of DSS in myocardial cells. (A) The protein expression of the Control, OGD, OGD + DSS-H, and OGD + DSS-H + ML385 groups detected by Western Blotting; (B) the histogram of these proteins ( $\mathrm{n}=4$, OGD vs. Control, ${ }^{* *} \mathrm{P}<0.01$; DSS-H vs. OGD, ${ }^{* \#} \mathrm{P}<0.01$; DSS-H + ML385 vs. DSS-H groups, ${ }^{* \&} \mathrm{P}<0.01$ ). DSS, $3{ }^{\prime}-$ daidzein sodium sulfonate; OGD, oxygen glucose deprivation; NRF2, nuclear factor erythroid 2-related factor 2; HO-1, Heme Oxygenase 1; L, low concentration; H, high concentration. 


\section{Footnote}

Reporting Checklist: The authors have completed the ARRIVE reporting checklist. Available at https://dx.doi. org/10.21037/jtd-21-1909

Data Sharing Statement: Available at https://dx.doi. org/10.21037/jtd-21-1909

Conflicts of Interest: All authors have completed the ICMJE uniform disclosure form (available at https://dx.doi. org/10.21037/jtd-21-1909). The authors have no conflicts of interest to declare.

Ethical Statement: The authors are accountable for all aspects of the work in ensuring that questions related to the accuracy or integrity of any part of the work are appropriately investigated and resolved. Experiments were performed under a project license (No. LLSC-2020090802) granted by Ethics Committee of The First Affiliated Hospital of Gannan Medical University, in compliance with institutional guidelines for the care and use of animals.

Open Access Statement: This is an Open Access article distributed in accordance with the Creative Commons Attribution-NonCommercial-NoDerivs 4.0 International License (CC BY-NC-ND 4.0), which permits the noncommercial replication and distribution of the article with the strict proviso that no changes or edits are made and the original work is properly cited (including links to both the formal publication through the relevant DOI and the license). See: https://creativecommons.org/licenses/by-nc-nd/4.0/.

\section{References}

1. Mensah GA, Roth GA, Fuster V. The Global Burden of Cardiovascular Diseases and Risk Factors: 2020 and Beyond. J Am Coll Cardiol 2019;74:2529-32.

2. Zhang T, Cheng G, Deng L, et al. Silence of S1 RNA binding domain 1 represses cell growth and promotes apoptosis in human non-small cell lung cancer cells. Transl Lung Cancer Res 2019;8:760-74.

3. Xu T, Ding W, Ji X, et al. Oxidative Stress in Cell Death and Cardiovascular Diseases. Oxid Med Cell Longev 2019;2019:9030563.

4. Aljakna A, Fracasso T, Sabatasso S. Molecular tissue changes in early myocardial ischemia: from pathophysiology to the identification of new diagnostic markers. Int J Legal Med 2018;132:425-38.

5. Kurian GA, Rajagopal R, Vedantham S, et al. The Role of Oxidative Stress in Myocardial Ischemia and Reperfusion Injury and Remodeling: Revisited. Oxid Med Cell Longev 2016;2016:1656450.

6. Di Filippo C, Cuzzocrea S, Rossi F, et al. Oxidative stress as the leading cause of acute myocardial infarction in diabetics. Cardiovasc Drug Rev 2006;24:77-87.

7. Neri M, Fineschi V, Di Paolo M, et al. Cardiac oxidative stress and inflammatory cytokines response after myocardial infarction. Curr Vasc Pharmacol 2015;13:26-36.

8. Moris D, Spartalis M, Spartalis E, et al. The role of reactive oxygen species in the pathophysiology of cardiovascular diseases and the clinical significance of myocardial redox. Ann Transl Med 2017;5:326.

9. Turer AT, Hill JA. Pathogenesis of myocardial ischemiareperfusion injury and rationale for therapy. Am J Cardiol 2010;106:360-8.

10. Krajka-Kuźniak V, Paluszczak J, Baer-Dubowska W. The Nrf2-ARE signaling pathway: An update on its regulation and possible role in cancer prevention and treatment. Pharmacol Rep 2017;69:393-402.

11. Ning H, Chen H, Deng J, et al. Exosomes secreted by FNDC5-BMMSCs protect myocardial infarction by antiinflammation and macrophage polarization via $\mathrm{NF}-\kappa \mathrm{B}$ signaling pathway and Nrf2/HO-1 axis. Stem Cell Res Ther 2021;12:519.

12. Yuan W, Chen Q, Zeng J, et al. 3'-Daidzein sulfonate sodium improves mitochondrial functions after cerebral ischemia/reperfusion injury. Neural Regen Res 2017;12:235-41.

13. Vieira JM, Norman S, Villa Del Campo C, et al. The cardiac lymphatic system stimulates resolution of inflammation following myocardial infarction. J Clin Invest 2018;128:3402-12.

14. Lu Z, Abe J, Taunton J, et al. Reactive oxygen speciesinduced activation of p90 ribosomal S6 kinase prolongs cardiac repolarization through inhibiting outward $\mathrm{K}+$ channel activity. Circ Res 2008;103:269-78.

15. Fouad Shalaby MA, Abd El Latif HA, El Yamani M, et al. Therapeutic activity of sarpogrelate and dopamine D2 receptor agonists on cardiovascular and renal systems in rats with alloxan-induced diabetes. BMC Pharmacol Toxicol 2021;22:64.

16. Li B, Wang Z, Yu M, et al. miR-22-3p enhances the intrinsic regenerative abilities of primary sensory neurons via the CBL/p-EGFR/p-STAT3/GAP43/p-GAP43 axis. J Cell Physiol 2020;235:4605-17. 
17. Li Y, Zou J, Li B, et al. Anticancer effects of melatonin via regulating lncRNA JPX-Wnt/ $\beta$-catenin signalling pathway in human osteosarcoma cells. J Cell Mol Med 2021;25:9543-56.

18. Ganguly S, Mitra A, Sarkar S. Role of $\alpha$-crystallin B in regulation of stress induced cardiomyocyte apoptosis. Cardiovasc Hematol Agents Med Chem 2014;12:60-5.

19. Pi SF, Liu YW, Li T, et al. Effect of sequential nicorandil on myocardial microcirculation and short-term prognosis in acute myocardial infarction patients undergoing coronary intervention. J Thorac Dis 2019;11:744-52.

20. Broughton KM, Wang BJ, Firouzi F, et al. Mechanisms of Cardiac Repair and Regeneration. Circ Res 2018;122:1151-63.

Cite this article as: Zeng $\mathrm{X}$, Yu J, Zeng T, Liu Y, Li B. 3 '-daidzein sulfonate protects myocardial cells from hypoxicischemic injury via the $\mathrm{NRF} 2 / \mathrm{HO}-1$ signaling pathway. J Thorac Dis 2021;13(12):6897-6910. doi: 10.21037/jtd-21-1909
21. Nitti M, Ivaldo C, Traverso N, et al. Clinical Significance of Heme Oxygenase 1 in Tumor Progression. Antioxidants (Basel) 2021;10:789.

22. Zhang X, Yu Y, Lei H, et al. The Nrf-2/HO-1 Signaling Axis: A Ray of Hope in Cardiovascular Diseases. Cardiol Res Pract 2020;2020:5695723.

23. Chen G, Liu G, Cao D, et al. Polydatin protects against acute myocardial infarction-induced cardiac damage by activation of Nrf2/HO-1 signaling. J Nat Med 2019;73:85-92.

24. Wallace DC. Mitochondrial diseases in man and mouse. Science 1999;283:1482-8.

(English Language Editor: L. Huleatt) 